\title{
碳/玻璃纤维混合编织网增强混凝土电热性能的 数值模拟与试验研究
}

\author{
徐世烺 ${ }^{(1)}$ ，尉文婷 (2)，宋世德 ${ }^{(3)}$ \\ (1) 浙江大学高性能建筑结构与材料研究所, 杭州 310058; \\ (2) 铁道第三勘察设计院集团有限公司, 天津 300251; \\ (3) 大连理工大学海岸与近海工程国家重点实验室, 大连 116024 \\ *E-mail: slxu@zju.edu.cn \\ 收稿日期: 2010-11-23; 接受日期: 2011-01-06 \\ 国家自然科学基金重点项目(批准号: 50438010)资助
}

\begin{abstract}
摘要碳/玻璃纤维混合编织网增强混凝土是一种新型纤维增强水泥基复合材料, 不 仅具有良好的力学性能, 而且具有优异的导电性能. 本文进行了碳/玻璃纤维混合编织 网增强混凝小板电热升温试验, 并建立数学模型, 采用有限元进行数值模拟, 得到了 不同环境温度、导热层厚度、输入功率条件下小板表面的温度变化规律, 并进行了有 无隔热层情况的对比. 结果表明: 碳/玻璃纤维混合编织网增强混凝土通电后可以形成 良好的导电加热网络, 产生热量使混凝土温度升高, 升温过程中电阻率十分稳定, 数 值分析结果与实验结果吻合较好. 通过野外融雪试验, 验证了该材料良好的电热融雪 效果. 利用碳/玻璃纤维混合编织网增强混凝土的电热性能, 可以实现安全、环保、高 效的融雪化冰.
\end{abstract}

关键词

碳/玻璃纤维混合编织网

数值模拟

电热性能

融雪化冰
在冬季, 水泥混凝土路面积雪结冰时, 会给道路 畅通和行车安全带来严重影响, 甚至导致高速公路 全线封闭或机场关闭, 给人民生活带来不便, 同时也 造成了巨大的经济损失 ${ }^{[1]}$. 因此, 亟需寻求一种安 全、经济、环保、高效的清除路面冰雪的方法, 保证 城市生命线的畅通.

目前国内外清除冰雪的方法主要有清除法和融 化法. 清除法包括人工清除和机械清除, 融化法包括 化学融化法和加热融化法. 机械清除往往清除不彻 底, 并且经济效益较差. 化学融雪法被许多国家和地 区广泛采用. 然而, 融雪剂的大量使用给路面结构和
环境带来了诸多负面效应, 如钢筋、钢纤维锈蚀, 路 面剥蚀破坏, 环境污染和植被破坏等 ${ }^{[2]}$. 加热融雪法 包括地热管法、红外线灯照加热法、电热丝法、太阳 能蓄热法、发热电缆加热法、导电混凝土加热法等 ${ }^{[3 \sim 9]}$. 导电混凝土是在普通混凝土中添加一定含量的导电 介质制成的水泥基复合材料，导电介质一般有钢渣、 钢纤维、碳纤维、碳质骨料、石墨、纳米炭黑等. 导 电混凝土加热法具有环保、高效等优点, 但其电阻率 易受到混凝土工作状态、电极接触电阻、外部环境等 诸多因素的影响，导致实际控制的不稳定.

本文提出利用碳/玻璃纤维混合编织网增强混凝

英文版发表信息: Xu S L, Yu W T, Song S D. Numerical simulation and experimental study on electrothermal properties of carbon/glass fiber hybrid textile reinforced concrete. Sci China Tech Sci, 2011, 54: 2421-2428, doi: 10.1007/s11431-011-4503-0 
土电热融雪的方法. 碳/玻璃纤维混合编织网是由纤 维粗纱编织而成的平面网状纺织物, 经向为碳纤维, 纬向为抗碱玻璃纤维. 碳纤维化学性能稳定, 具有高 抗拉强度、高弹性模量以及优异的导电性能 ${ }^{[10]}$. 将碳 /玻璃纤维混合编织网铺设在混凝土中, 利用碳纤维 束的高导电性, 玻璃纤维束的定向作用, 将碳纤维束 按一定方式彼此连接, 便可以形成稳定的导电体系, 通电后迅速产生热量使混凝土温度升高, 在保证低 能耗的情况下得到最佳的融雪化冰效果, 并能通过纤 维编织网对混凝土起到增强、阻裂作用, 延长使用寿 命 ${ }^{[11]}$. 本文主要从数值模拟和试验两方面对碳/玻璃 纤维混合编织网增强混凝土的电热性能进行分析, 研究环境温度、输入功率、导热层厚度对升温规律的 影响, 观察升温过程中电阻稳定性, 并通过野外融雪 试验验证其应用价值.

\section{1 数值研究}

\section{1 计算模型}

将碳/玻璃纤维混合编织网铺设在混凝土中, 碳 纤维束通电后将产生热量, 使混凝土内部温度上升, 当表面温度达到 $0^{\circ} \mathrm{C}$ 以上时, 冰雪便会吸热升温, 最 终融化. 对于热传导的数值分析, 即在一定的边界条 件和控制微分方程下, 计算得到物体内部的温度分 布. 本问题属于非稳态热传导计算问题.

为简化问题，作以下合理假定.

(i) 考虑纤维束长度与纤维束间距相比很大, 温 度场在沿碳纤维束方向上变化很小, 故可以忽略该 方向的传热.

(ii) 除边缘的碳纤维束, 其他碳纤维束之间的分 布基本相同, 以两碳纤维束中间断面为对称, 可以看 成周期性结构模型.

(iii) 研究区域内均为均质恒物性材料.

(iv) 不考虑接触热阻.

因此, 传热过程可以看作二维非稳态有内热源 导热问题 ${ }^{[12]}$. 简化后的计算模型如图 1 所示.

控制微分方程

$$
\frac{\partial T}{\partial t}=\frac{\lambda}{\rho c}\left(\frac{\partial T^{2}}{\partial x^{2}}+\frac{\partial T^{2}}{\partial y^{2}}\right)+\frac{\stackrel{q_{v}}{\rho c}}{\rho c},
$$

式中, $T$ 为温度, $t$ 为时间, $\rho$ 为混凝土的密度, $\lambda$ 为导热 系数, $c$ 为比热, $q_{v}$ 为内热源强度.
初始条件

$$
\left.T\right|_{t=0}=T_{0} .
$$

边界条件

(i) 碳/玻璃纤维混合编织网增强混凝土板四周
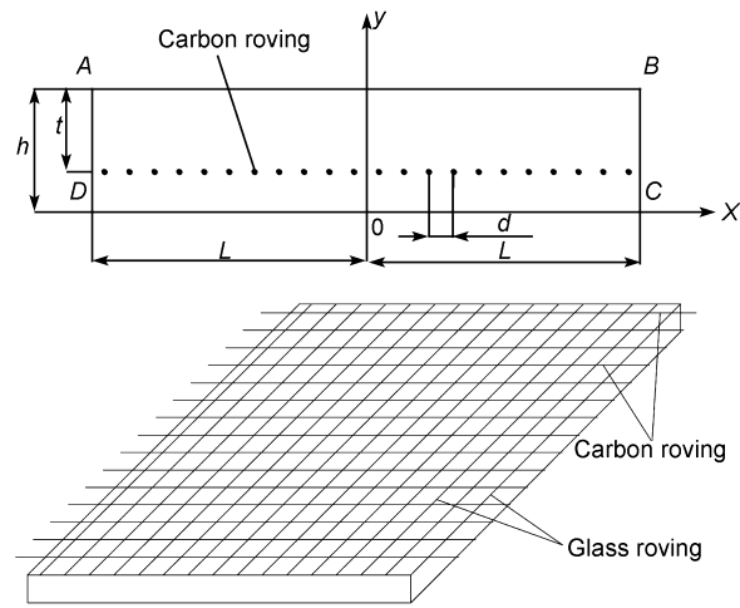

图 1 计算模型

和底面近似认为绝热. 由于板内温度场的对称性, 对 每一个计算单元, 沿着 $x$ 轴的 2 个表面可近似认为绝 热

$$
\begin{gathered}
\left.\frac{\partial T}{\partial x}\right|_{x=-L}=\left.\frac{\partial T}{\partial x}\right|_{x=L}=0, \\
\left.\frac{\partial T}{\partial y}\right|_{y=0}=0 .
\end{gathered}
$$

(ii) 假定碳纤维束截面形状为圆形, 近似认为其 内部温度均匀分布, 并与表面温度相同. 将碳纤维束 的线功率折算成热流密度, 作为碳纤维束表面的热 荷载

$$
q_{0}=\frac{q_{l}}{\pi d},
$$

式中, $q_{0}$ 为碳纤维束表面的热流通量, $q_{l}$ 为线功率, $d$ 为碳纤维束的直径.

其中, 碳纤维束的直径 $d$

$$
d=\sqrt{\frac{4 A}{\pi}}=\sqrt{\frac{4 T e x}{\pi \rho}},
$$

式中, $A$ 为碳纤维束的理论面积, $T e x$ 为碳纤维束单 位长度的质量, $\rho$ 为碳纤维束的密度.

(iii) 板的上表面与空气存在对流换热和辐射换 
热

$$
\left.\lambda \frac{\partial T}{\partial y}\right|_{y=h}=-h\left(T-T_{f}\right),
$$

式中, $\lambda$ 为导热系数, $h$ 为综合换热系数, $T_{f}$ 为环境温 度.

\section{2 有限元分析前处理}

在构建模型时，单元类型选用 4 节点四边形单元 PLANE55, 按照表 1 定义材料属性, 由图 1 建立实体 模型. 其中, 模型长度 $L=200 \mathrm{~mm}$, 高度 $h=40 \mathrm{~mm}$, 导 热层厚度 $t=30 \mathrm{~mm}$, 碳纤维间距 $d=10 \mathrm{~mm}$.

整个计算区域采用不均分网格划分. 网格划分 结果如图 2: 单元数 2354 个, 节点数 2442 个.

\section{3 有限元计算结果分析}

任取一种工况计算. 设环境温度为 $-16.6^{\circ} \mathrm{C}$, 加 热功率 $1200 \mathrm{~W} / \mathrm{m}^{2}$, 折算成热荷载为 $6369 \mathrm{~W} / \mathrm{m}^{2}$. 设 定荷载步持续时间为 $36000 \mathrm{~s}$, 共划分了 600 个荷载 步, 时间步长为 $60 \mathrm{~s}$. 图 3 给出了各不同加热时间段 结构内部的温度场分布. 温度由碳纤维束至其两侧 的混凝土层逐渐递减, 并且越接近结构层表面, 温度 梯度越小, 结构内部及表面温度分布十分均匀. 由于 施加了绝热条件, 下表面的温升速率要明显高于上 表面.

由图 4 可见, 模型的上表面中心温度最初上升较 快, 随着导热过程的进行, 由于结构层内部蓄热, 温 升速率减慢, 温度曲线渐趋平缓, 最终温度接近 $120^{\circ} \mathrm{C}$ 保持恒定.

\section{2 原料及试件制作}

\section{1 原材料}

试验采用碳/玻璃纤维混合编织网, 经向为碳纤 维, 纬向为耐碱玻璃纤维, 网格尺寸为 $10 \mathrm{~mm} \times 10$ $\mathrm{mm}$. 纤维性能指标见表 2.

基体采用 P.O42.5R 普通硅酸盐水泥、I 级粉煤 灰、硅灰、河砂(最大粒径 $1.2 \mathrm{~mm}$ )、水和超塑化剂. 为 保证混凝土基体充分渗透过纤维编织网, 与其形成 的良好黏结, 避免孔穴, 混凝土基体需要具有优良的 工作性能(匀质、稳定、抗离析等). 采用粉煤灰、硅 灰作为掺和料可满足基体高流动性、低干缩的要求 ${ }^{[13]}$.
基体的成分和配比见表 3.

\section{表 1 材料热物性参数}

\begin{tabular}{cccc}
\hline Material & $\begin{array}{c}\text { Thermal } \\
\text { conductivity } \\
\left(\mathrm{W} /\left(\mathrm{m} \cdot{ }^{\circ} \mathrm{C}\right)\right)\end{array}$ & $\begin{array}{c}\text { Specific heat } \\
\left(\mathrm{J} /\left(\mathrm{kg} \cdot{ }^{\circ} \mathrm{C}\right)\right)\end{array}$ & $\begin{array}{c}\text { Density } \\
\left(\mathrm{kg} / \mathrm{m}^{3}\right)\end{array}$ \\
\hline Concrete & 2.34 & 1046 & 2100 \\
\hline
\end{tabular}

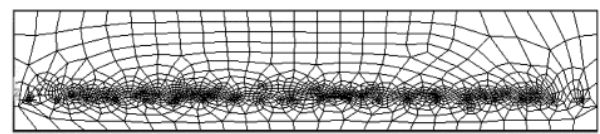

图 2 网格划分结果

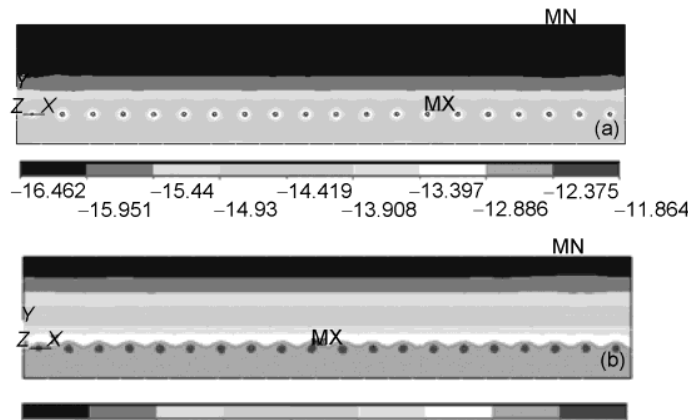

$8.93110 .299^{11.666_{13.033^{14.4}} 15.768^{17.135_{18.502}{ }^{19.869}} 21.237}$

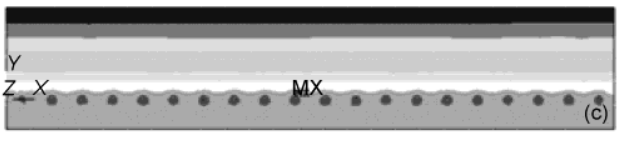

$31.97733 .604^{35.231} 36.050^{38.486} 40.113^{41.74} 43.367^{44.995} 46.622$

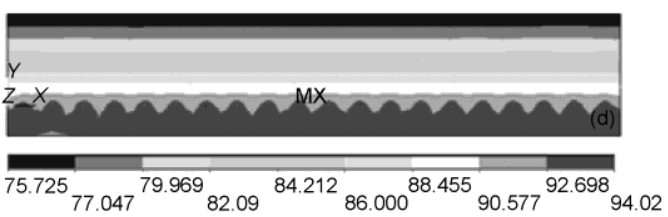

图 3 结构内部温度场分布

(a) $1 \mathrm{~min}$; (b) $30 \mathrm{~min}$; (c) $60 \mathrm{~min}$; (d) $150 \mathrm{~min}$

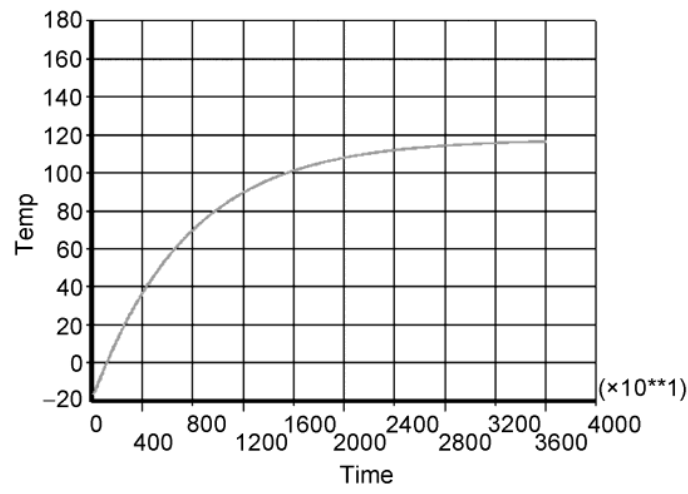

图 4 板表面中心温度随时间的变化关系曲线 
表 2 纤维材料性能

\begin{tabular}{cccccccc}
\hline Roving & Type (k) & Tensile strength (MPa) & Tensile modulus (GPa) & Ultimate strain $(\%)$ & Tex $(\mathrm{g} / \mathrm{km})$ & Density $\left(\mathrm{g} / \mathrm{cm}^{3}\right)$ & Resistivity $(\mu \Omega \cdot \mathrm{m})$ \\
\hline Carbon & 12 & 4900 & 230 & 2.1 & 800 & 1.8 & 22 \\
AR-glass & 6 & 3500 & 74 & 4.7 & 600 & 2.57 & - \\
\hline
\end{tabular}

\section{表 3 基体的配比组成}

\begin{tabular}{cc}
\hline Component & Content $\left(\mathrm{kg} / \mathrm{m}^{3}\right)$ \\
\hline Portland cement 42.5R & 375 \\
Fly ash & 115 \\
Silica fume & 20 \\
Water & 204 \\
Super plasticizer & 17 \\
Sand 0-0.6 mm & 562 \\
Sand 0.6-1.2 mm & 1124 \\
\hline
\end{tabular}

\section{2 试件制作}

由于纤维束由大量纤维单丝组成，埋入混凝土 中后, 混凝土不能完全浸入到纤维束内部, 只有最外 层纤维丝能与混凝土形成较好黏结, 大大降低了纤 维编织网的协调受力能力. 试验前将纤维编织网采 用环氧树脂浸渍处理, 环氧树脂使渗透到纤维束内 部, 固化后可将纤维束结成一体, 提高内外层纤维丝 的协同受力能力 ${ }^{[14]}$. 在环氧树脂固化前对纤维网进 行粘砂处理, 如图 5 所示, 可以进一步提高纤维束和 基体的黏结能力 ${ }^{[15]}$.

按设计配比将水泥、粉煤灰、砂、硅灰放入搅拌 机内搅拌 3 5 min, 然后加入水、减水剂继续摚拌 5 min. 在 $400 \mathrm{~mm} \times 400 \mathrm{~mm} \times 40 \mathrm{~mm}$ 的木模具中首先浇 注厚度为 $30 \mathrm{~mm}$ 的混凝土层, 轻微振捣抹平, 然后铺 上处理完毕的纤维编织网, 最后浇注 $10 \mathrm{~mm}$ 厚的混 凝土层并振捣抹平. $24 \mathrm{~h}$ 后拆模, 在标准条件下养护 28 天.

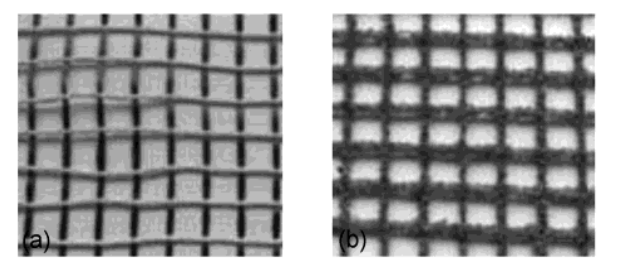

图 5 纤维网表面处理示意图

(a)处理前;(b)环氧浸渍并粘砂

\section{3 试验设备}

试验设备采用 $24 \mathrm{~V} / 15 \mathrm{~A}$ 直流稳压电源, 数字万 用表(UT71D), 铂电阻温度传感器(Pt100), 数字测温 仪.

\section{3 电热升温试验}

\section{1 碳纤维单束电阻}

将试件两侧的碳纤维束表面处理干净, 接入导 线. 采用 $3 / 4$ 精度的数字万用表测量试件中单束碳纤 维束的电阻. 由于制作误差, 纤维编织网网格间距并 不严格符合 $10 \mathrm{~mm} \times 10 \mathrm{~mm}$, 从而试件中总共包含 32 束碳纤维束，单束纤维电阻值列于表 4. 由表 4 可见， 碳纤维单束电阻值大部分较为接近, 个别电阻值较 大, 是由于纤维束内部缺陷或接触电阻引起的, 平均 电阻值为 $24.5 \Omega$. 将 32 束碳纤维束全部并联, 总的 电阻值约为 $0.74 \Omega$; 全部串联电阻值约为 $784.06 \Omega$. 因而, 通过改变碳纤维束的接线方式可以实现试件的

\section{表 4 碳纤维单束电阻}

\begin{tabular}{|c|c|c|c|c|c|c|c|c|}
\hline No. of roving & 1 & 2 & 3 & 4 & 5 & 6 & 7 & 8 \\
\hline Resistance $(\Omega)$ & 21.59 & 23.02 & 26.38 & 23.72 & 28.59 & 21.43 & 36.47 & 19.2 \\
\hline No. of roving & 9 & 10 & 11 & 12 & 13 & 14 & 15 & 16 \\
\hline Resistance $(\Omega)$ & 25.83 & 22.99 & 28.26 & 20.44 & 28.51 & 20.26 & 21.46 & 29.38 \\
\hline No. of roving & 17 & 18 & 19 & 20 & 21 & 22 & 23 & 24 \\
\hline Resistance $(\Omega)$ & 20.59 & 38.74 & 25.18 & 31.74 & 27.23 & 28.65 & 25.58 & 18.71 \\
\hline No. of roving & 25 & 26 & 27 & 28 & 29 & 30 & 31 & 32 \\
\hline Resistance $(\Omega)$ & 20.5 & 20.79 & 20.77 & 20.46 & 24.33 & 21.03 & 21.36 & 20.87 \\
\hline
\end{tabular}




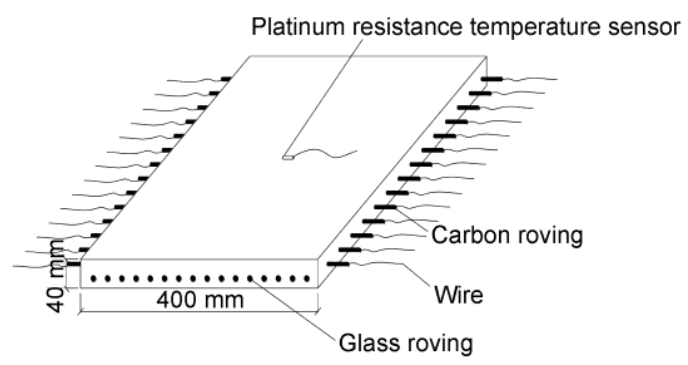

图 6 碳/玻璃纤维混合编织网增强混凝土试件

电阻值在 $0.74 \sim 784.06 \Omega$ 之间分段变化，从而使 加热功率在一定范围内控制可调.

\section{2 电热升温试验}

将小板两侧的 32 根碳纤维束接入导线, 在纤维 与导线的连接处做好电绝缘, 然后将碳纤维束按串、 并联相结合的方式连接, 将铂电阻传感器固定在小 板上表面的中心, 底面和侧面包裹 $30 \mathrm{~mm}$ 厚聚苯乙 烯泡沫塑料来减少热损失, 如图 6. 将小板放入冰柜, 调整冰柜温度至试验设定的温度, 经过 $24 \mathrm{~h}$, 小板内 的温度可与冰柜环境温度达到一致. 然后将小板接 入电路, 接通电源, 小板产生热量, 温度上升. 在通 电加热过程中观察并记录试件温度及电阻率的变化.

为了灵活调节加热功率, 采用 PWM 控制技术, 利用微处理器的数字输出来对电路进行功率控制, 分析不同输入功率条件下的板的升温规律. 测试原 理如图 7.

板的加热等效电阻率和电加热功率分别按下式 计算

$$
\begin{gathered}
\rho=\frac{R A}{L}=\frac{V A}{I L}, \\
P=\frac{U^{2}}{R}=\frac{U^{2} A}{\rho L},
\end{gathered}
$$

式中, $\rho$ 板的加热等效电阻率, $R$ 为板的加热等效电阻, $L$ 为板沿碳纤维束方向的长度, $A$ 为板垂直于碳纤维 束方向的截面面积, $V$ 为输入电压, $I$ 为电流.

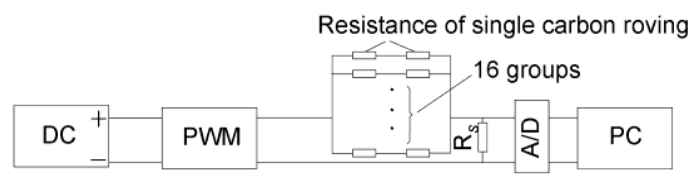

图 7 PWM 控制装置

\section{3 数值模拟与试验结果对比与讨论}

图 8 给出了输入功率 $1300 \mathrm{~W} / \mathrm{m}^{2}$, 不同环境温度 下, 10 和 $30 \mathrm{~mm}$ 两种导热层厚度试件的表面温度变 化曲线. 小板上表面温度随着时间的增长而逐渐升 高, 初期温度上升较快, 随后温升速率逐渐减慢, 趋 于稳定. 各工况的温升曲线趋势基本一致.

由图 8(a), 导热层厚度为 $30 \mathrm{~mm}$, 环境温度为 $-16.6^{\circ} \mathrm{C}$ 时, 小板通电 $150 \mathrm{~min}$ 后温度达到 $71.5^{\circ} \mathrm{C}$, 平 均升温速率为 $0.59^{\circ} \mathrm{C} / \mathrm{min}$; 对于初始温度为 $-8.1^{\circ} \mathrm{C}$ 的 工况, 小板通电 $150 \mathrm{~min}$ 后温度达到 $74.2^{\circ} \mathrm{C}$, 温度曲 线上升趋势与 $-16.6^{\circ} \mathrm{C}$ 工况下基本一致. 由于小板本 身的电阻率较小, 在 $24 \mathrm{~V}$ 电压下便可获得较大的发 热功率, 从而环境温度未对温升造成显著的影响.

图 8(b)为导热层厚度 $10 \mathrm{~mm}$ 情况下小板表面的 升温曲线. 在环境温度为 $-16.6^{\circ} \mathrm{C}$, 小板通电 $150 \mathrm{~min}$ 后温度达到 $68.9^{\circ} \mathrm{C}$, 平均升温速率为 $0.57^{\circ} \mathrm{C} / \mathrm{min}$; 在 初始温度为 $-10.2^{\circ} \mathrm{C}, 150 \mathrm{~min}$ 后板面温度达到 $70.4^{\circ} \mathrm{C}$.

图 8(c)比较了同一环境温度, 相同输入功率, 不 同导热层厚度的温度变化曲线. 两条温升曲线基本 重合, 即在电加热功率较大的情况下, 导热层厚度对 温升的影响不明显. 考虑使用环境、低能耗、耐久性 等因素，可将导热层厚度控制在 10 50 mm.

由图 8(a)和(b)数值计算与试验结果的对比曲线 可见, 两者在初始一段时间吻合较好, 随着导热过程 的进行, 数值计算结果要偏高于实验结果, 这与实验 过程中仍存在热损失有一定的关系.

在试验过程中, 同时对小板电阻的变化进行了 观测. 在 $150 \mathrm{~min}$ 加热过程中, 导热层厚度为 $30 \mathrm{~mm}$ 的试件, 整体加热电阻率由 $-16.6^{\circ} \mathrm{C}$ 的 $10.21 \Omega \cdot \mathrm{cm}$ 下 降到 $71.5^{\circ} \mathrm{C}$ 的 $9.75 \Omega \cdot \mathrm{cm}$, 降幅 $4.5 \%$; 在另一工况下, 整体加热电阻率由 $-8.1^{\circ} \mathrm{C}$ 的 $10.01 \Omega \cdot \mathrm{cm}$ 下降到 $74.2^{\circ} \mathrm{C}$ 的 $9.63 \Omega \cdot \mathrm{cm}$, 降幅 $3.8 \%$, 如图 9(a). 对于导热 层厚度为 $10 \mathrm{~mm}$ 的试件, 试件温度由 $-16.6^{\circ} \mathrm{C}$ 上升到 $68.9^{\circ} \mathrm{C}$ 过程中, 试件的整体电阻率由 $10.89 \Omega \cdot \mathrm{cm}$ 下 降到 $10.5 \Omega \cdot \mathrm{cm}$, 降幅 $3.58 \%$; 在另一工况下, 试件温 度由 $-10.2^{\circ} \mathrm{C}$ 上升到 $70.4^{\circ} \mathrm{C}$ 过程中, 试件的整体电阻 率由 $10.81 \Omega \cdot \mathrm{cm}$ 下降到 $10.37 \Omega \cdot \mathrm{cm}$, 降幅为 $4.07 \%$, 如图 9(b). 四次升温/降温循环中, 四次升温/降温循 环中, 电阻率的变化幅度很小, 且基本一致. 因此, 碳/玻璃纤维混合编织网增强混凝土的电阻率十分稳 定，在实际应用中能实现稳定的温度控制.

图 10 给出了不同电加热功率条件下, 有无隔热 

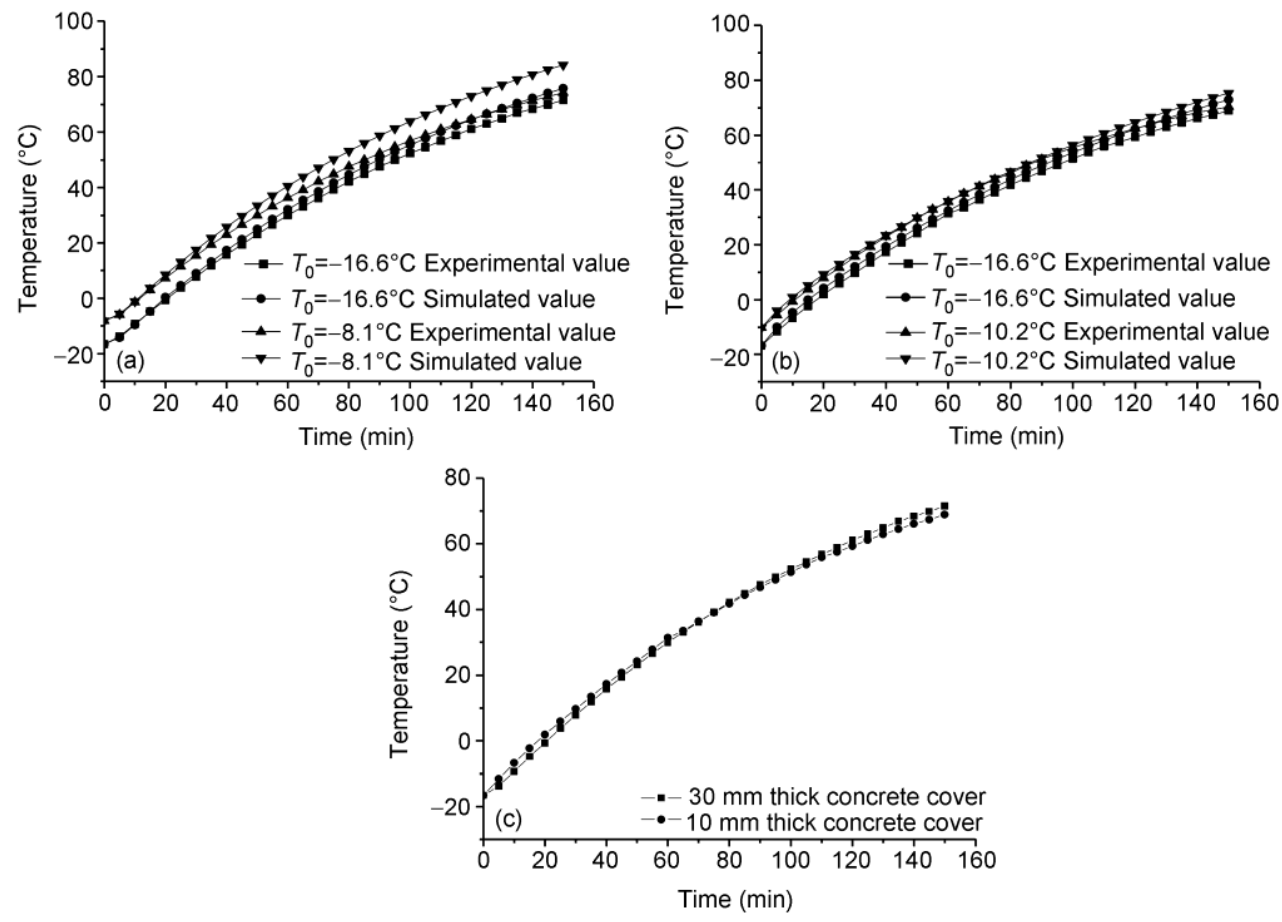

图 8 不同导热层厚度温度随时间的变化关系曲线

(a) $t=30 \mathrm{~mm}$; (b) $t=10 \mathrm{~mm}$; (c) 两种导热层厚度的升温关系对比
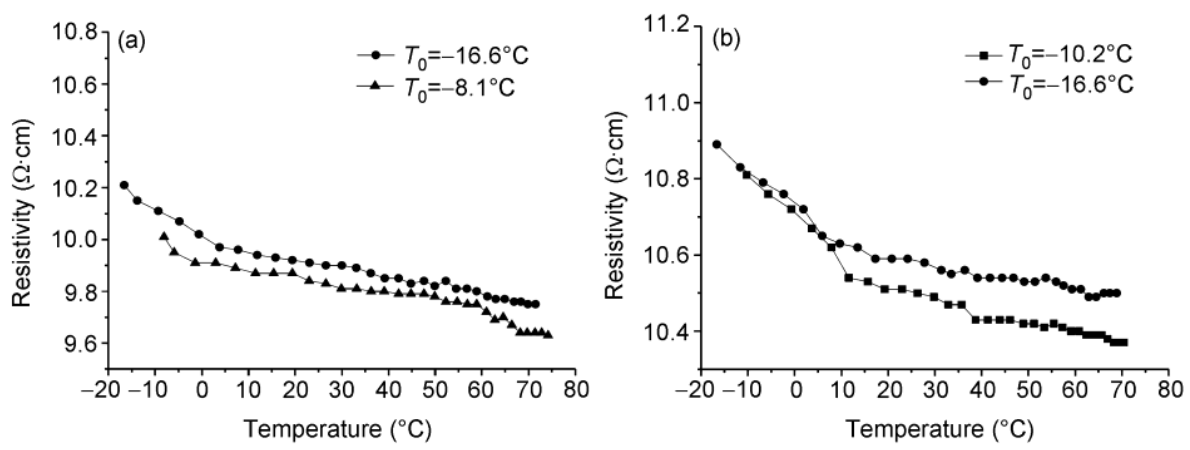

图 9 不同导热层厚度电阻率随温度的变化关系曲线

(a) $t=30 \mathrm{~mm}$; (b) $t=10 \mathrm{~mm}$
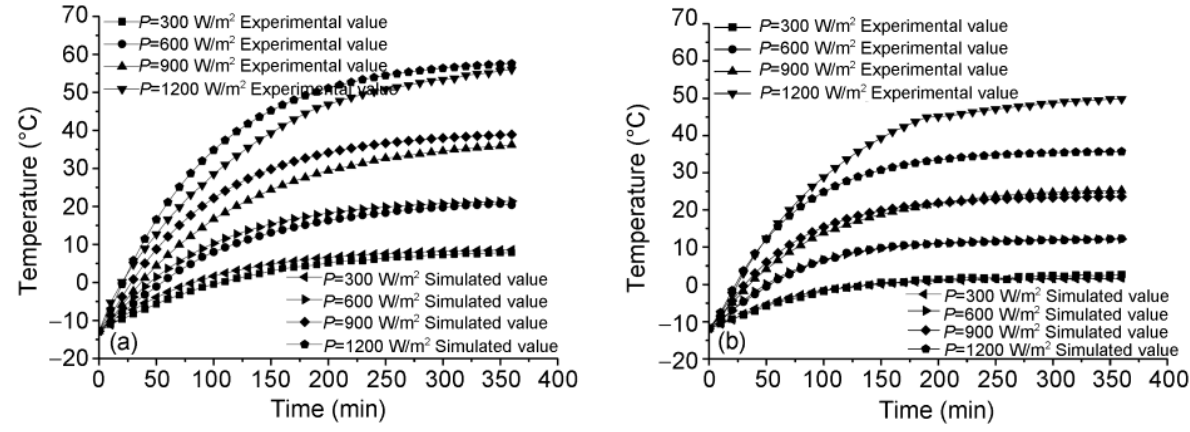

图 10 有、无隔热层情况下温度随时间的变化关系曲线 $(t=\mathbf{3 0} \mathrm{mm})$

(a) 有隔热层; (b) 无隔热层 
层两种工况试件表面温度变化规律. 从图 10 可以看 出, 输入电功率越大, 单位时间内板产生的热量越 多, 升温速率越快. 通电 $6 \mathrm{~h}$ 后, $300 \mathrm{~W} / \mathrm{m}^{2}$ 功率下有 隔热层情况下板表面所达到的稳态温度是无隔热层 情况下的 2.9 倍, $600 \mathrm{~W} / \mathrm{m}^{2}$ 功率下为 1.7 倍, $900 \mathrm{~W} / \mathrm{m}^{2}$ 功率下为 1.4 倍, $1200 \mathrm{~W} / \mathrm{m}^{2}$ 功率下为 1.1 倍, 即加热 电功率越小, 隔热层的效果越明显. 因此, 在发热层 底部设置隔热层对于减少能量损耗、降低工程应用成 本是十分必要的.

通过对比相应的数值模拟结果与试验结果, 二 者部分吻合较好, 对于存在的部分差异主要由于如 下原因.

(i) 数值模拟假定试验材料为均质恒物性材料, 而实际试件材料的密度、比热、导热系数均会随配比、 密实度、含水率、温度等因素变化.

(ii) 数值模拟所选用的对流换热系数为定值, 而 实验过程中环境变化会引起对流换热系数的变化, 使试件上表面与空气间的对流换热发生变化.

(iii)碳纤维束接线位置聚苯乙烯泡沫塑料无法包 裹严密, 存在一定热量损失, 使得试验值较模拟值偏 低, 这种影响会随着试件尺寸的增大而减弱.
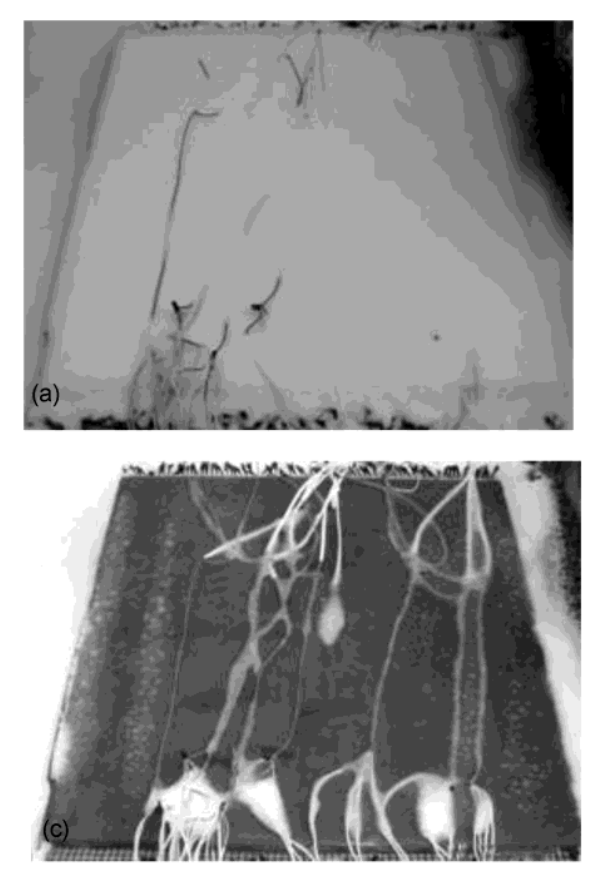

图 11 实时融雪试验过程

（a) 初始状态; (b) 通电 $15 \mathrm{~min}$ 后; (c) 通电 $30 \mathrm{~min}$ 后; (d) 通电 $45 \mathrm{~min}$ 后

\section{4 野外融雪试验}

制作尺寸 $1000 \mathrm{~mm} \times 950 \mathrm{~mm} \times 40 \mathrm{~mm}$ 的碳/玻璃纤 维混合编织网增强混凝土大板, 在 2009 年 2 月 18 日 进行了野外实时融雪试验. 环境温度 $-1^{\circ} \mathrm{C} \sim-2^{\circ} \mathrm{C}$, 东 南风 2 3 级, 降雪等级为中雪. 将碳/玻璃纤维混合编 织网增强混凝土大板放置在自然环境中, 底未设隔 热层. 凌晨开始降雪, 早晨 9 点开始进行融雪试验. 板表面初始积雪厚度约 $30 \mathrm{~mm}$. 大板碳纤维束全部 并联接 $24 \mathrm{~V}$ 直流稳压电源进行通电融雪, 加热功率 $900 \mathrm{~W} / \mathrm{m}^{2}$.

由图 11 可见, 通电 $15 \mathrm{~min}$ 后大板表面已经有部 分积雪开始融化, $30 \mathrm{~min}$ 后积雪基本化完, $45 \mathrm{~min}$ 后 积雪已完全融化, 雪降落在板上即时融化, 无任何积 雪积水现象. 由图 12 可以看出, 开始加热至 $40 \mathrm{~min}$ 融雪过程中, 板面温度上升缓慢, 平均升温速率 $0.26^{\circ} \mathrm{C} / \mathrm{min} ; 40 \mathrm{~min}$ 后温度迅速上升, 平均升温速率 $0.74^{\circ} \mathrm{C} / \mathrm{min}$, 说明雪已经完全融化. 由图 13 可见, 由 开始通电至 $25 \mathrm{~min}$ 后, 板的电阻率由 $2.503 \Omega \cdot \mathrm{cm}$ 下 降到 $2.443 \Omega \cdot \mathrm{cm}$ 降幅为 $2.4 \%$; 之后直到通电 $60 \mathrm{~min}$ 后, 大板的电阻率保持在 $2.44 \Omega \cdot \mathrm{cm}$ 左右, 十分稳定. 由此可见, 利用碳/玻璃纤维混合编织网增强混凝土
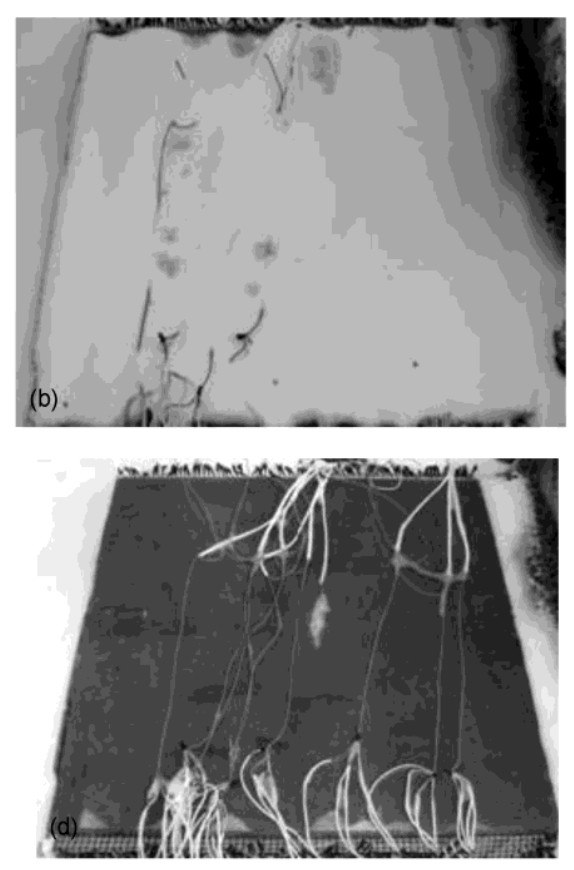


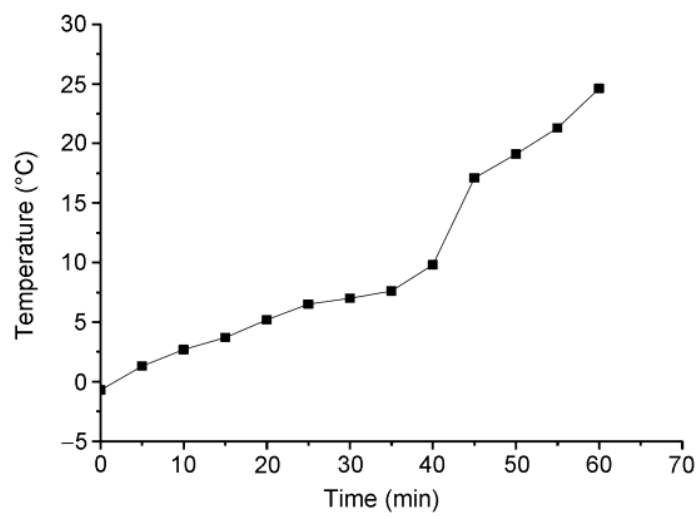

图 12 温度随时间的变化

电热融雪可以取得较好的效果.

\section{5 结论}

本文主要对碳/玻璃纤维混合编织网增强混凝土 小板进行了电热升温试验和数值模拟研究. 通过改 变碳纤维束之间的接线方式能够使碳/玻璃纤维混合 编织网增强混凝土的等效电阻在较大范围内变化, 从而可以方便灵活控制输入功率; 在较低的安全电 压下便可实现较大发热功率迅速升温; 并且升温过

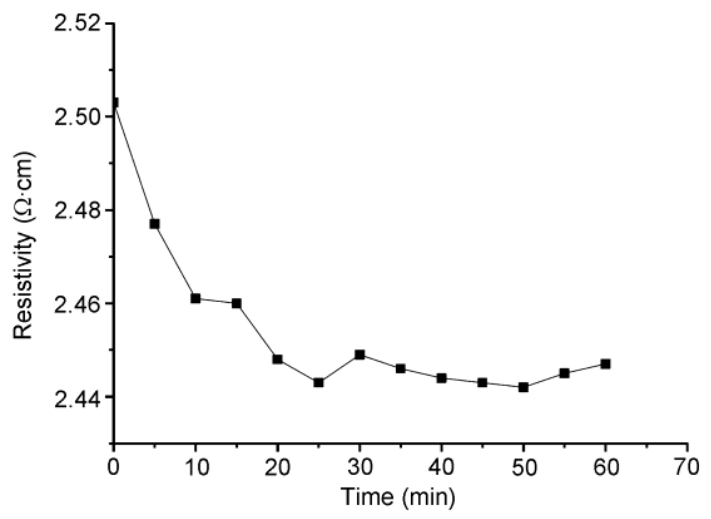

图 13 电阻率随时间的变化

程中电阻率十分稳定，具有良好的电热性能. 设置隔 热层, 尤其在较低加热功率情况下, 能有效减少能量 损耗. 通过合理假设简化数学计算模型，采用非线性 有限元方法进行数值模拟分析，探测结构内部的温 度分布与变化, 得到的模拟结果与试验结果基本吻 合. 大板野外融雪试验表明利用碳/玻璃纤维混合编 织网增强混凝土电热融雪可以取得良好的效果, 验 证了其工程应用价值. 在实际应用过程中，碳/玻璃 纤维混合编织网增强混凝土用于不同环境下融雪化 冰的智能控制有待进一步研究.

\section{参考文献}

1 Sherif Y, Christopher Y T. Conductive concrete overlay for bridge deck deicing. ACI Mater J, 1999, 96: 382390

2 洪乃丰. 撒盐融化冰雪的利弊与趋势分析. 工业建筑, 2008, 38: 82-84

3 Sherif Y, Christopher Y T. Conductive concrete overlay for bridge deck deicing. Mix design, optimization, and properties. ACI Mater J, 2000, 97: 172-181

4 李炎锋, 武海琴, 王贯明, 等. 发热电缆用于路面融雪化冰的实验研究. 北京工业大学学报, 2006, 32: 217-222

5 高青，于鸣，刘小兵. 基于蓄能的道路热融雪化冰技术及其分析. 公路, 2007,(5): 170-174

6 李丹, 董发勤, 沈刚. 钢纤维石墨导电混凝土在路面除冰雪中的应用研究. 建筑门窗与金属建材, 2004, (11): 61-64

7 许孝春, 宋建成, 冯晋阳. 钢渣-碳纤维发热混凝土地板的研制. 混凝土, 2008, (2): 122-124

8 唐祖全, 李卓球, 钱觉时. 碳纤维导电混凝土在路面除冰雪中的应用研究. 建筑材料学报, 2004, 7: 215-220

9 王小英, 孙明清, 侯作富, 等. 纳米炭黑水泥砂浆的导电性与电热特性研究. 功能材料, 2006, (11): 1841-1847

10 Xu G D, Hannant D J. F1exural behaviour of combined polypropylene network and glass fiber reinforced cement. Cement Concr Compos, 1992, 14: 51-61

11 徐世烺, 尹世平. 纤维编织网联合钢筋增强混凝土梁受弯性能解析理论. 中国科学：技术科学, 2010, 40: 619-629

12 杨世铭, 陶文铨. 传热学. 北京: 高等教育出版社, 2006

13 徐世烺, 李赫. 碳纤维编织网和高性能细粒混凝土的粘结性能. 建筑材料学报, 2006, 9: 211-215

14 Xu S L, Markus K, Hans-Wolf R. Bond characteristics of carbon, alkali-resistant glass and aramid textiles in mortar. ASCE J Mater Civ Eng, 2004, 16: 356-364

15 徐世烺, Hans-Wolf R, Markus K. 高性能精细混凝土与碳纤维织物粘结性能研究. 见：第十一届全国结构工程学术会议论文集, 2002. 95-104 